\title{
Focus point from direct gauge mediation
}

\author{
Sibo Zheng ${ }^{\mathrm{a}}$ \\ Department of Physics, Chongqing University, Chongqing 401331, People's Republic of China
}

Received: 22 September 2014 / Accepted: 8 December 2014 / Published online: 14 January 2015

(C) The Author(s) 2014. This article is published with open access at Springerlink.com

\begin{abstract}
This paperis devoted to the reconciliation of the tension between the theoretic expectation from naturalness and the present LHC limits on the superpartner mass bounds. We argue that in supersymmetry models of direct gauge mediation the focusing phenomenon appears, which dramatically reduces the fine tuning associated to the $126 \mathrm{GeV}$ Higgs boson. This type of model is highly predictive as regards the mass spectrum, with a multi-TeV third generation, the $A_{t}$ term of order $1 \mathrm{TeV}$, gluino mass above LHC mass bound, and the light neutralinos and charginos below $1 \mathrm{TeV}$.
\end{abstract}

\section{Introduction}

As the LHC keeps running, the searches of supersymmetry (SUSY) signals such as stop/gluino, bottom, and Higgs mass discovered at $126 \mathrm{GeV}[1,2]$ continue to push their mass bounds toward the multi-TeV range $[3,4]$. On the other hand, the argument of naturalness requires the masses of third generation scalars, the Higgsinos, and gluinos should be $\sim 1 \mathrm{TeV}$. This is the present status of SUSY.

To reconcile the experimental limits and the expectation of naturalness, either of them needs subtle reconsiderations. In this paper, we consider relaxing the upper bounds from the argument of naturalness. The upper bounds on the above soft breaking parameters arise from the significant contribution to the renormalization group (RG) running for up-type Higgs mass squared $m_{H_{\mu}}^{2}$, which connects to the electroweak (EW) scale through the electroweak symmetry-breaking (EWSB) condition [for $\tan \beta>10$ in the context of the minimal supersymmetric model (MSSM)],

$m_{Z}^{2} \simeq-2 \mu^{2}-2 m_{H_{\mu}}^{2}$.

Naively, low fine tuning implies that the value of $\mu$ and $\left|m_{H_{\mu}}\right|$ at EW scale should be both near EW scale. But there exists an exception. In some cases, there is significantly

a e-mail: sibozheng.zju@gmail.com cancelation among the RGE corrections arising from soft breaking parameters to $m_{H_{\mu}}^{2}$, although their input values are far beyond $1 \mathrm{TeV}$. This is known as the focusing phenomenon $[5,6]$.

The early attempts in [5-8] were mainly restricted to SUSY models near the grand unification scale (GUT). One recent work related to focus point SUSY deals with gaugino mediation [9]. In this text, we consider gauge mediated (GM) SUSY models with intermediate or low messenger scale $M$ (for a review see, e.g., [10]). Since the focusing phenomenon can be analytically estimated only if the gaugino masses dominate over all other soft breaking masses, or if they are small compared with the thirdgeneration scalar masses (with [11] or without [5,6] $A$ terms), following this observation, in this paper we study a direct GM model, in which the gaugino masses are naturally small due to the fact that gaugino masses of order $\mathcal{O}(F)$ vanish [12].

Another rationale for employing direct GM models is that the focusing phenomenon can be understood as a result of hidden symmetry. This is so because, without directly gauging the global symmetries of the model, there would be larger symmetries maintained in the hidden theory. Otherwise, without the protection of symmetry a tiny deviation for the model parameters from their focus point values leads to significant fine tuning again, and the model is actually unnatural.

As we will see, there are three free input parameters in our model. Two of them are fixed so as to induce the focusing phenomenon, leaving an overall mass parameter $m_{0}$. The fit to the $126 \mathrm{GeV}$ Higgs boson discovered at the LHC then determines the magnitude of this parameter, with $m_{0} \sim 4$ $7 \mathrm{TeV}$. Thus, our model is highly predictive as regards the mass spectrum.

In Sect. 2.1, we introduce the model in detail. In Sect. 2.2, we discuss the focusing phenomenon, the boundary conditions for such structure and the mass spectrum at EW scale. In Sect. 2.3, we discuss the possibility of uplifting thegluino 
mass above the LHC lower bound while keeping the focusing. Finally we conclude in Sect. 3.

\section{The model}

\subsection{Setup}

In contrast to [13], in which a non-minimal GM model was employed to discuss focusing phenomenon, we study SUSY models that do not spoil the grand unification of SM gauge couplings and are restricted to the context of direct GM. The messenger fields include chiral quark superfields $q+q^{\prime}$ and their bi-fundamental fields $\bar{q}+\bar{q}^{\prime}$, lepton superfields $l+$ $l^{\prime}$ and their bi-fundamental fields $\bar{l}+\bar{l}^{\prime}$, and singlet $S$ and its bi-fundamental field $\bar{S}$. They transform under $S U(3)_{C} \times$ $S U(2)_{L} \times U(1)_{Y}$ as, respectively,

$$
\begin{gathered}
q, q^{\prime} \sim\left(\mathbf{3}, \mathbf{1},-\frac{1}{3}\right), \\
\bar{q}, \overline{q^{\prime}} \sim\left(\overline{\mathbf{3}}, \mathbf{1}, \frac{1}{3}\right), \\
l, l^{\prime} \sim\left(\mathbf{1}, \mathbf{2}, \frac{1}{2}\right), \\
\bar{l}, \overline{l^{\prime}} \sim\left(\mathbf{1}, \overline{\mathbf{2}},-\frac{1}{2}\right),
\end{gathered}
$$$$
S, \bar{S} \sim(\mathbf{1}, \mathbf{1}, 0)
$$

So, these messenger multiplets complete a $\mathbf{5}+\overline{\mathbf{5}}$ representation of the SM gauge group. The renormalizable superpotential consistent with the SM gauge symmetry is given by ${ }^{1}$

$W=f X+X q \bar{q}+X l \bar{l}+m\left(q^{\prime} \bar{q}+q \bar{q}^{\prime}\right)+m\left(l^{\prime} \bar{l}+l \bar{l}^{\prime}\right)$,

where $X=M+F \theta^{2}$, denotes the SUSY-breaking sector with nonzero $F$ term. We will consider $N$ copies of such messengers multiplets, with $N<6$ so as to maintain the grand unification of SM gauge couplings.

For the purpose of focusing we add a deformation to the superpotential Eq. (3),

$W=\lambda H_{u} S \bar{l}$.

This superpotential can be argued to be natural by either imposing a hidden $U(1)_{X}$ symmetry [15] or matter parity [16]. For example, we can impose the $U(1)_{X}$ charges of the fields,

$q_{X}\left(X, \phi_{i}, \bar{\phi}_{i}, H_{u}, H_{d}\right)=(1,-1 / 2,-1 / 2,1,-1)$

\footnotetext{
${ }^{1}$ It belongs to the general Wess-Zumino model, which can be completed as an effective theory of strong dynamics at low energy [14]. The direct gauge mediation arises after gauging the global symmetries in the weak theory and identifying them as SM gauge groups.
}

where $\phi_{i}=\left\{q, q^{\prime}, l, l^{\prime}, S\right\}$. In addition, this hidden symmetry forbids some operators such as $H_{d} S l$.

In Eq. (3) we have assumed a unified mass parameter $m$ and ignored the Yukawa coefficients for simplicity. For $m<M$, which we adopt, in this paper the soft scalar mass spectrum induced by superpotential Eq. (3) is the same as that of the minimal GM at the leading order. Since the minimal GM cannot induce the focusing phenomenon, the deformation to the scalar mass spectrum due to Eq. (4) is crucial for our purpose. In particular, Eq. (4) gives rise to a negative one-loop contribution to $m_{H_{u}}^{2}$ with suppression factor $F / M^{2}$. Unless we take $\sqrt{F}<<M$, the sign of $m_{H_{u}}^{2}$ would be negative, it will not lead to focusing (see explanation around Eq. (11)). Therefore, we are restricted to choosing

$m<M$, and $\sqrt{F}<<M$.

For a detailed calculation of the deviation to the scalar mass spectrum given by Eq. (4), we refer the reader to [15, 17]. With small SUSY breaking given by Eq. (6), $m_{H_{\mu}}^{2}$ will be uplifted as required for focusing.

One can verify that the gaugino masses at one loop of order $\mathcal{O}(F)$ vanish due to the fact the mass matrix of the messengers,

$\mathcal{M}=\left(\begin{array}{ll}X & m \\ m & 0\end{array}\right)$

satisfies $\operatorname{det} \mathcal{M}=$ const as long as $m$ does not vanish, although $m$ is small in comparison with the scale $M$. So we expect that the RGE for $m_{H_{\mu}}^{2}$ is dominated by the stop mass squared $m_{Q_{3}}^{2}, m_{u_{3}}^{2}$, and an induced A term (see Eq. (4)).

\subsection{Focusing and mass spectrum}

Following the observation $[5,6,11]$ that the REGs for $A_{t}$ and scalar masses such as $m_{H_{\mu}}^{2}$ are affected by both themselves and the gluino masses, while the RGE for gluino mass is only affected by itself, we can solve the RGEs for the soft scalar masses,

$$
\begin{gathered}
\left(\begin{array}{c}
m_{H_{\mu}}^{2}(Q) \\
m_{u_{3}}^{2}(Q) \\
m_{Q_{3}}^{2}(Q) \\
A_{t}^{2}(Q)
\end{array}\right)=\kappa_{12} I^{2}(Q)\left(\begin{array}{l}
3 \\
2 \\
1 \\
6
\end{array}\right)+\kappa_{6} I(Q)\left(\begin{array}{l}
3 \\
2 \\
1 \\
0
\end{array}\right) \\
+\kappa_{0}\left(\begin{array}{c}
1 \\
0 \\
-1 \\
0
\end{array}\right)+\kappa_{0}^{\prime}\left(\begin{array}{c}
0 \\
1 \\
-1 \\
0
\end{array}\right)
\end{gathered}
$$

for small gluino masses (to be compared with the above scalar soft masses). Here,

$I(Q)=\exp \left(\int_{\ln M}^{\ln Q} \frac{6 y_{t}^{2}\left(Q^{\prime}\right)}{8 \pi^{2}} \mathrm{~d} \ln Q^{\prime}\right)$, 


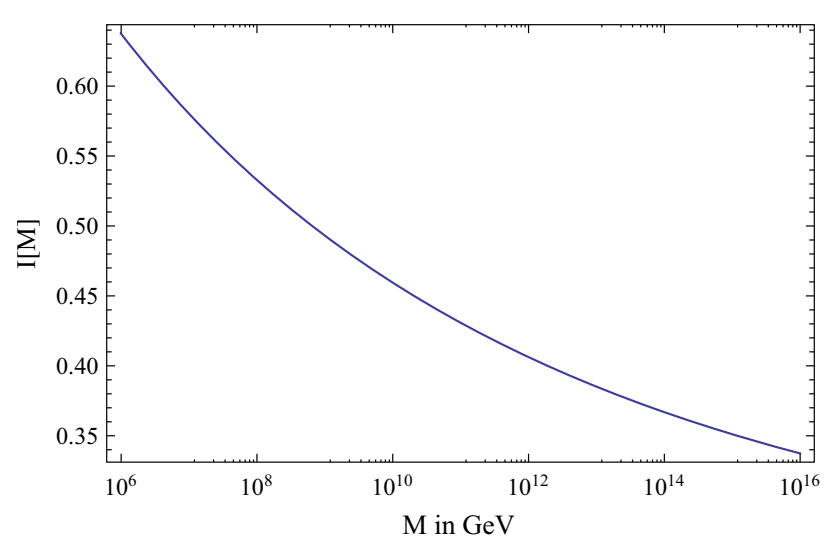

Fig. $1 I$ as a function of $M$ in the context of MSSM below the scale M

which depends on $M$ and the RGE for the top Yukawa. In Fig. 1 we show the numerical value of $I$ as a function of $M$, in the context of MSSM below the scale $M$. In particular, $I(1 \mathrm{TeV}) \simeq 0.527$ for $M=10^{8} \mathrm{GeV}$.

The condition for the focusing phenomenon can be derived from Eq. (8) by imposing $m_{H_{\mu}}^{2}(1 \mathrm{TeV}) \simeq 0$. Define $m_{H_{\mu}}^{2}(M)=+m_{0}^{2}$. Similar to [11] we choose $x$ to parameterize the splitting between $m_{Q_{3}}^{2}(M)$ and $m_{u_{3}}^{2}(M)$ and $y$ to be directly related to $A_{t}(M)$. In the case of small SUSY breaking, the mass spectrum which induces focusing at the scale $\mu=1 \mathrm{TeV}$ reads

$m_{0}^{2}\left(\begin{array}{c}1 \\ 1.41+x-1.58 y \\ 1.82-x-3.16 y \\ 9 y\end{array}\right)_{M} \rightarrow m_{0}^{2}\left(\begin{array}{c}0 \\ 0.74+x-1.58 y \\ 1.48-x-3.16 y \\ 1.66 y\end{array}\right)_{\mu}$.

Alternatively we rescale the parameter $x$ as in [11] such that $m_{Q_{3}}^{2}$ only depends on $x$. For $m_{H_{\mu}}^{2}(M)=-m_{0}^{2}$, Eq. (10) is instead

$m_{0}^{2}\left(\begin{array}{c}-1 \\ -1.41+x-1.58 y \\ -1.82-x-3.16 y \\ 9 y\end{array}\right)_{M} \rightarrow m_{0}^{2}\left(\begin{array}{c}0 \\ -0.74+x-1.58 y \\ -1.48-x-3.16 y \\ 1.66 y\end{array}\right)$.

This parameterization appears when $F / M^{2} \rightarrow 1$. In this limit, $m_{H_{\mu}}^{2}$ is dominated by the one-loop negative contribution proportional to the Yukawa coupling $\lambda$. From Eq. (11), there is no consistent solution to $x$ and $y$ in this case.

Soft masses in Eq. (8) at the scale $\mu=1 \mathrm{TeV}$ are functions of the Yukawa coupling $\lambda$, the number of messenger pairs $N$, the ratio $F / M^{2}$ and the SUSY-breaking mediated scale $M$. By Eq. (10) one connects the variables $(x, y)$ and the model parameters $\lambda$ and $N$. For the three input parameters $m_{0}, x$, and $y$ (with $M$ fixed) for focusing in the model, two of them can be fixed by the choices of $\lambda$ and $N$. We choose $x$ and $y$ for our analysis. Figure 2 shows the plots of $x$ (dotted) and

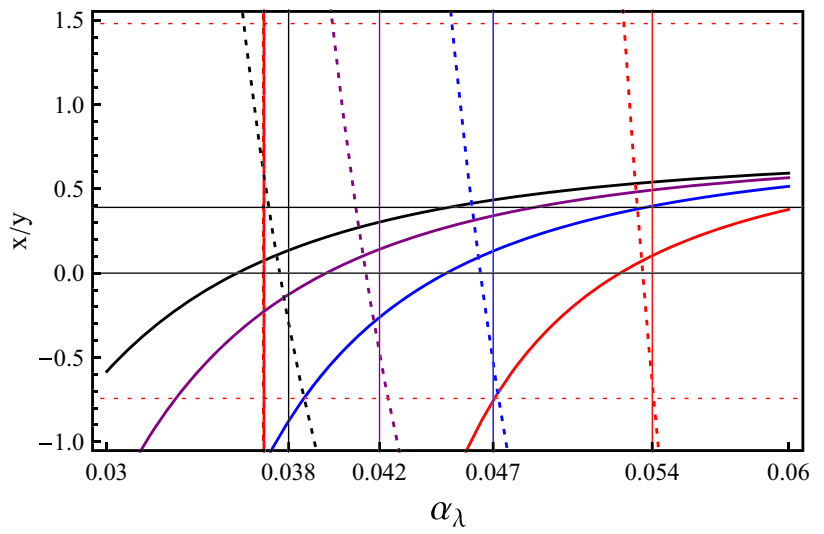

Fig. 2 Plots of $x$ (dotted) and $y$ (solid) as a function of $\alpha_{\lambda}$ for $N=$ $\{1,2,3,4\}$. The red, blue, purple, and black curves correspond to $N=$ $1,2,3,4$, respectively. For each $N$ the focus point values are read from the crossing points between vertical line and solid curve for $y$ and dotted curve for $x$, respectively. The dotted (solid) horizontal lines refer to the range allowed for $x(y)$

$y$ (solid) as a function of $\alpha_{\lambda}$ and $N$. For each $N$ the focus point values of $x$ and $y$ are read off from the crossing points between vertical line and solid curve (dotted curve) for $y(x)$. Therefore, there is only one free parameter left in the model by imposing the focusing condition, which is very predictive as regards the mass spectrum and signal analysis.

Since we perform our analysis in perturbative theory, in order to avoid the Landau pole up to GUT scale, the Yukawa coupling $\alpha_{\lambda}$ is upper bounded, $\sim 0.1$ for our choice of messenger scale. The dotted and solid horizontal lines in Fig. 2 refer to the allowed ranges for $x$ and $y$, respectively. These ranges are derived from the requirement that the stop soft masses are not tachyon-like and the $A_{t}$ squared is positive. Therefore, we obtain

$0<y<0.40,-0.74<x<1.48$,

$1.58 y-0.74<x<1.48-3.16 y$,

$1.58 y-1.41<x<1.82-3.16 y$.

It is easy to verify that for each $N$ the crossing points satisfy the constraints above.

With the focusing phenomenon we have a single free parameter at hand, namely $m_{0}$. It can be uniquely determined in terms of the mass of the Higgs boson observed at the LHC. Figure 2 shows how $m_{h}$ changes with the parameter $m_{0}$ for different Ns. The two-loop level Higgs boson mass in the MSSM is given by [18]

$$
\begin{aligned}
m_{h}^{2}= & m_{Z}^{2} \cos ^{2} 2 \beta+\frac{3 m_{t}^{4}}{4 \pi^{2} v^{2}}\left\{\log \left(\frac{M_{S}^{2}}{m_{t}^{2}}\right)+\frac{1}{2} \tilde{A}_{t}+\frac{1}{16 \pi^{2}}\right. \\
& \left.\times\left(\frac{3}{2} \frac{m_{t}^{2}}{v^{2}}-32 \pi \alpha_{3}\right)\left[\tilde{A}_{t}+\log \left(\frac{M_{S}^{2}}{m_{t}^{2}}\right)\right] \log \left(\frac{M_{S}^{2}}{m_{t}^{2}}\right)\right\} .
\end{aligned}
$$




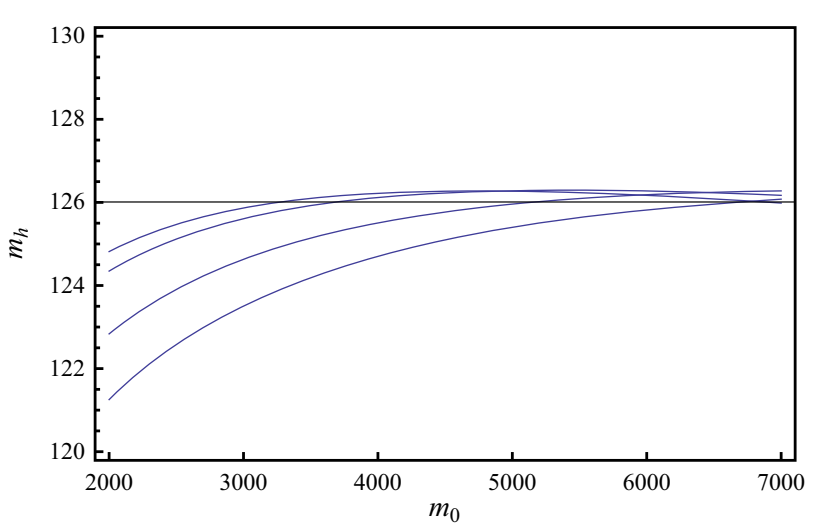

Fig. $3 m_{h}$ vs. $m_{0}$ for different $N$ s, with $N=1,2,3,4$ from bottom to top, respectively. A multi- $\mathrm{TeV} m_{0}$ is required by the $126 \mathrm{GeV}$ Higgs boson

Table 1 Given a focus point, the input mass parameter $m_{0}$ (in unit of $\mathrm{TeV}$ ) required for $m_{h}=126 \mathrm{GeV}$, and the corresponding soft mass spectrum (in unit of $\mathrm{TeV}$ ) at the renormalization scale $\mu=1 \mathrm{TeV}$ in the context of MSSM, for different values of the messenger number $N$

\begin{tabular}{lllll}
\hline & $N=1$ & $N=2$ & $N=3$ & $N=4$ \\
\hline$m_{0}$ & 7.0 & 5.9 & 4.0 & 3.5 \\
$m_{\tilde{t}_{1}}$ & 3.12 & 3.62 & 4.54 & 4.83 \\
$m_{\tilde{t}_{2}}$ & 7.65 & 4.98 & 4.80 & 6.0 \\
$A_{t}$ & 1.64 & 1.48 & 1.50 & 1.50 \\
$\mu$ & 0.50 & 0.42 & 0.28 & 0.24 \\
\hline
\end{tabular}

Here $v=174 \mathrm{GeV}$ and $\tilde{A}_{t}=\frac{2 X_{t}^{2}}{M_{S}^{2}}\left(1-\frac{X_{t}^{2}}{12 M_{S}^{2}}\right)$, with $X_{t}=A_{t}-\mu \cot \beta$. We focus on the large $\tan \beta$ region. For $\tan \beta \geq 20$, the fit to the Higgs boson mass does not change much. From Fig. 3 one observes that $m_{0} \sim 4.0-7.0$ due to the fit to the $126 \mathrm{GeV}$ Higgs boson.

Substituting the values of $m_{0}$ from Fig. 3 and $x, y$ from Fig. 2 into Eq. (10) we find the mass spectrum, which is shown in Table 1.

The choice on large $\tan \beta$ might be forbidden by a possibly large $\mathrm{B} \mu$ term induced by Eq. (4). As noted in [16], $\mathrm{B} \mu \sim \mu A_{t}$. In terms of the electroweak symmetry-breaking condition, we have $\sin (2 \beta) \simeq \mathrm{B} \mu / m_{0}^{2} \sim\left(A_{t} / m_{0}\right)^{2} \cdot\left(\mu / A_{t}\right)$. With a small $\mu$ term of order $\sim 300-500 \mathrm{GeV}$ (as shown in Table 1) at the messenger scale $M$, one does not have to worry about $\mu$ being made very large by a radiative correction involving heavy soft scalar masses (see, e.g., [17]). So, one obtains $\sin (2 \beta)$ of order $\sim(1 / 4)^{2} \cdot(1 / 4)$ from Table 1 , and the choice of a large value of $\tan \beta$ is not violated by the $\mathrm{B} \mu$ term.

\subsection{Gaugino mass}

As mentioned above due to $\operatorname{det} \mathcal{M}=$ const the gaugino masses vanish at one-loop level of order $\mathcal{O}(F)$ and at the two-loop level of order $\mathcal{O}(F)$. Their leading contributions appear at one-loop level of order $\mathcal{O}\left(F^{3} / M^{5}\right)$ [12]. In the small SUSY-breaking limit the magnitude of the gaugino mass relative to $m_{Q_{3}}$ at the input scale is given by ${ }^{2}$

$$
\frac{m_{\tilde{g}_{i}}}{m_{Q_{3}}} \sim\left(\frac{F}{M^{2}}\right)^{2} \cdot \frac{\sqrt{N} \alpha_{i}}{\sqrt{2 \times\left(\frac{4}{3} \alpha_{3}^{2}(M)+\frac{3}{4} \alpha_{2}^{2}(M)+\frac{1}{60} \alpha_{1}^{2}(M)\right)}} .
$$

Using one-loop RGEs for the gluino masses, we find their values at the renormalization scale $\mu=1 \mathrm{TeV}$. One observes from Eq. (13) that the gluino mass is far below the 2013 LHC bound $\simeq 1.3 \mathrm{TeV}$ due to the suppression by a factor $F^{2} / M^{4}$.

Without extra significant modifications to the gaugino mass spectrum, the LHC bound would exclude this simple model, despite it providing a natural explanation of the Higgs boson mass and being consistent with the present experimental limits. Here, we propose a recipe [19] in terms of imposing a small modification to the superpotential $\delta W=$ $m^{\prime}\left(\bar{l}^{\prime} l^{\prime}+\bar{q}^{\prime} q^{\prime}\right)$, with a small mass $m^{\prime}<m$. These mass terms are consistent with the gauge symmetries and matter parity of the messenger sector.

If so, Eq. (7) will be instead

$\mathcal{M}=\left(\begin{array}{ll}X & m \\ m & m^{\prime}\end{array}\right)$

The correction to the soft scalar mass spectrum is of the order $\mathcal{O}\left(\mathrm{m}^{\prime 4} / \mathrm{m}^{4}\right)$ and very weak. However, the correction to the gaugino mass, which is of order

$m_{\tilde{g}_{i}} \simeq N \cdot \frac{\alpha_{i}}{4 \pi} \cdot \frac{F}{m} \cdot \frac{m^{\prime}}{m}$,

can be large enough to reconcile with the LHC bound when $\mathrm{m}^{\prime} / \mathrm{m}$ is larger than $F^{2} / M^{4}$. For example, we choose $N=1$, $M=10^{8} \mathrm{GeV}$ and $m=0.1 M$. Then $m_{0} \sim 7 \mathrm{TeV}$ and $\sqrt{F} \sim 8.2 \times 10^{6} \mathrm{GeV}$, and further $m_{\tilde{g}_{3}} \sim 7 \times 10^{-3} \times m^{\prime}$ from Eq. (15). The LHC gluino mass bound requires $m^{\prime} \geq$ $2 \times 10^{5} \mathrm{GeV}$, which is consistent with the constraint $m^{\prime}<$ $m<M$. The bino and wino masses are both near $1 \mathrm{TeV}$. So they are the main target of $14-\mathrm{TeV}$ LHC.

\section{Discussion}

As shown in the mass spectrum of Table 1, the main source for fine tuning arises from the $\mu$ term and the gluino mass $M_{\tilde{g}_{3}}$. The fine tuning parameter $c$, which is defined as $c=\max \left\{c_{i}\right\}$, with

$c_{i}=\left|\frac{\partial \ln m_{Z}^{2}}{\partial \ln a_{i}}\right|$

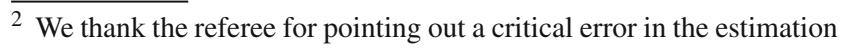
of the gaugino mass in the previous version of this manuscript. 
where the $a_{i}$ are the soft mass parameters involved, is given by $c=\max \left\{c_{\mu}, c_{M_{\tilde{g}_{3}}}\right\}$. We find that $c=c_{M_{\tilde{g}_{3}}} \sim 200$ for $M=10^{8} \mathrm{GeV}$ and the electroweak value $M_{\tilde{g}_{3}}=1.3 \mathrm{TeV}$ by using the gluino mass induced RG correction to $\delta m_{H_{u}}^{2}$.

As for other indirect experimental limits, such as flavor changing neutral violation, the model feels comfortable. This is because the masses of the three-generation sleptons and first two-generation squarks are all of order $\sim$ multi-TeV, with a high degeneracy in each sector.

What about the sensitivity of our results to the messenger scale? At first, the assumption that there exists a completion of strong dynamics at high energy indicates that $M$ should be smaller than the GUT scale. Typically, we have $M<$ $10^{10} \mathrm{GeV}$ in the context of direct gauge mediation. For the case of low-scale mediation, i.e., $M<10^{8} \mathrm{GeV}$, the gluino mass is already close to the 2013 LHC mass bound. In other words, $M=10^{8} \mathrm{GeV}$, as we studied in detail, is a reference value for intermediate-scale SUSY model. The promising signals for this simple and natural model include searching gluinos, neutralinos, and charginos at the LHC.

Along this line it is of interest to extend the modelindependent focusing condition to the whole energy range below the GUT scale [20], and construct natural SUSY models in the context of either direct or non-direct GM.

Acknowledgments The work is supported in part by the Natural Science Foundation of China under grant No. 11247031 and 11405015.

Open Access This article is distributed under the terms of the Creative Commons Attribution License which permits any use, distribution, and reproduction in any medium, provided the original author(s) and the source are credited.

Funded by SCOAP $^{3}$ / License Version CC BY 4.0.

\section{References}

1. ATLAS Collaboration, Phys. Lett. B 710, 49 (2012). arXiv:1202.1408 [hep-ex]

2. The CMS Collaboration, Phys. Lett. B 710, 26 (2012). arXiv:1202.1488 [hep-ex]

3. ATLAS Collaboration, ATLAS SUSY 2013 stop summary. https:// twiki.cern.ch/twiki/pub/AtlasPublic/CombinedSummaryPlots/ ATLAS_directstop_all_SUSY2013.pdf

4. CMS Collaboration, CMS SUSY 2013 stop summary. https://twiki. cern.ch/twiki/pub/CMSPublic/SUSYSMSSummaryPlots8TeV/ SUSY2013T2ttT6.pdf

5. J.L. Feng, K.T. Matchev, T. Moroi, Phys. Rev. Lett. 84, 2322 (2000)

6. J.L. Feng, K.T. Matchev, T. Moroi, Phys. Rev. D 61, 075005 (2000)

7. F. Brummer, W. Buchmuller, JHEP 1205, 006 (2012). arXiv:1201.4338 [hep-ph]

8. F. Brmmer, M. Ibe, T.T. Yanagida, Phys. Lett. B 726, 364 (2013). arXiv:1303.1622 [hep-ph]

9. T. Yanagida, N. Yokozaki, Phys. Lett. B 722, 355 (2013)

10. G.F. Giudice, R. Rattazzi, Phys. Rep. 322, 419 (1999)

11. J.L. Feng, D. Sanford, Phys. Rev. D86, 055015 (2012)

12. K.-I. Izawa, Y. Nomura, K. Tobe, T. Yanagida, Phys. Rev. D 56, 2886 (1997)

13. K. Agashe, Phys. Rev. D 61, 115006 (2000)

14. K.A. Intriligator, N. Seiberg, D. Shih, JHEP 04, 021 (2006)

15. N. Craig, S. Knapen, D. Shih, Y. Zhao, JHEP 1303, 154 (2013). arXiv:1206.4086 [hep-ph]

16. K. Hamaguchi, N. Yokozaki, Phys. Lett. B 694, 398 (2011). arXiv:1007.3323 [hep-ph]

17. S. Zheng, Eur. Phys. J. C 74, 2724 (2014). arXiv:1308.5377 [hep$\mathrm{ph}]$

18. M. Carena, J.R. Espinosa, M. Quiros, C.E.M. Wagner, Phys. Lett. B 355, 209 (1995)

19. R. Kitano, H. Ooguri, Y. Ookouchi, Phys. Rev. D 75, 045022 (2007). hep-ph/0612139

20. S. Zheng et al., to appear (2015) 